\title{
Commentary on 'Surgical interventions for posterior compartment prolapse and obstructed defecation symptoms: a systematic review with clinical practice recommendations'
}

\author{
Yu Hwee $\operatorname{Tan}^{1}$ \\ Received: 15 June 2019 / Accepted: 15 July 2019 / Published online: 29 July 2019 \\ (C) The International Urogynecological Association 2019
}

This systematic review by Grimes et al. looks at the impact of different surgical interventions in the posterior compartment on prolapse and defecatory symptoms, assessing both anatomic and symptom outcomes for different surgical approaches. Given the wide variety of surgical techniques available to address the posterior compartment, the aim of the review was to identify the best evidence-based surgical approach to address posterior vaginal wall prolapse in patients with symptomatic obstructed defecation [1].

The authors conducted literature searches of MEDLINE, Embase and ClinicalTrials.gov from inception to April 4, 2018. Randomized controlled trials, prospective and retrospective comparative and single-group studies of women undergoing posterior vaginal compartment surgery for vaginal bulge or bowel symptoms were included. Outcomes assessed included pre- and postoperative anatomic measures of prolapse and symptom measures of obstructed defecation. Forty-six eligible studies reported on six surgery types including laparoscopic sacrocolpoperineopexy, graft augmentation, site-specific defect repair, native tissue posterior colporrhaphy, transanal approach and stapled transanal rectal resection (STARR), with analyses categorized into the six surgery types and three comparisons between surgeries (native tissue posterior colporrhaphy versus biologic graft augmentation, native tissue posterior colporrhaphy versus site-specific defect repair, native tissue posterior colporrhaphy versus transanal approach). The clinical recommendation suggested by the review was that for women with rectocele and obstructed defecation symptoms requiring surgical intervention, traditional transvaginal native-tissue posterior colporrhaphy should be considered first to improve the anatomy

Yu Hwee Tan

hwee85@gmail.com

1 Department of Urogynecology, Varsity Lakes Day Hospital, Gold Coast, QLD, Australia and symptoms of obstructed defecation albeit with a higher risk of dyspareunia relative to other surgical methods. This recommendation is supported by a previous review that provided grade A evidence that the transvaginal approach to the posterior compartment is superior to the transanal approach in the treatment of prolapse [2].

This concise systematic review provides a good summary of the evidence available for the surgical methods used for posterior compartment prolapse and obstructed defecation and provides an answer to the clinical question posed. However, the clinician must bear in mind that there may be insufficient evidence for the other surgical techniques, given the highest quality data available are for native tissue posterior colporrhaphy and the three comparisons performed were all against native tissue posterior colporrhaphy. A further limitation is the heterogeneity in the way anatomic and symptom outcomes were assessed in the included studies. Grimes et al. provide useful guidance for the way we can surgically manage posterior compartment prolapse and obstructed defecation; however, as the review states, further comparative long-term studies to support this would be welcome.

\section{Compliance with ethical standards}

Conflicts of interest None.

\section{References}

1. Grimes CL, Schimpf MO, Wieslander CK, et al. Surgical interventions for posterior compartment prolapse and obstructed defecation symptoms: a systematic review with clinical practice recommendations. Int Urogyn J. 2019. https://doi.org/10.1007/s00192-019-04001-z.

2. Karram M, Maher C. Surgery for posterior vaginal wall prolapse. Int Urogyn J. 2013;24(11):1835-41.

Publisher's note Springer Nature remains neutral with regard to jurisdictional claims in published maps and institutional affiliations. 